\title{
Efficacy of Rapid Decongestion Strategy in Patients Hospitalized for Acute Heart Failure
}

\author{
Mitsutoshi Oguri, MD, PhD; Hideki Ishii, MD, PhD; Kunihiko Takahara, MD; \\ Kenichiro Yasuda, MD; Tomonobu Takikawa, MD; Takuya Sumi, MD, PhD; \\ Hiroshi Takahashi, BSc; Toyoaki Murohara, MD, PhD
}

\begin{abstract}
Background: Clinical congestion is the most dominant feature in patients with acute decompensated heart failure (HF). However, uncertainty exists due to the permutations and combinations of congestion status and decongestion strategies. This study investigated the effect of congestion status and its improvement on 1-year mortality.

Methods and Results: In all, 453 consecutive patients hospitalized for acute decompensated HF between July 2015 and March 2017 were prospectively included in the study. Congestion was evaluated using the congestion score. The 1-year mortality rate was $22.7 \%$. The mean ( \pm SD) congestion scores at admission, on Day 3, and at discharge were 10.7 $\pm 3.9,3.4 \pm 3.5$, and $0.3 \pm 0.8$, respectively. The improvement rate in congestion scores during the first 3 days was $78 \% ; 46.6 \%$ of patients had residual congestion. The Day 3 congestion score and the improvement rate during the first 3 days were related to 1 -year all-cause mortality and cardiovascular mortality. Combined predictive values were examined by calculating multivariable-adjusted hazard ratios for associations of residual congestion and improvement rate during the first 3 days, and prognostic variables identified by the Cox regression model. Residual congestion and lesser improvement $(<64 \%)$ were associated with higher relative risk of 1 -year all-cause mortality and cardiovascular mortality than residual congestion and higher improvement $(\geq 64 \%)$ or resolved congestion.
\end{abstract}

Conclusions: Rapid decongestion could be a prerequisite regardless of residual congestion in hospitalized acute decompensated HF patients.

Key Words: Decongestion; Heart failure; Prognosis

$\mathbf{T}$ he incidence and prevalence of patients with heart failure (HF) is steadily increasing along with advancing age in Japan.1,2 Regardless of recent advances in the control of HF, including medical treatments and device therapies, HF is still a grievous clinical problem due to high mortality and rehospitalization rates. ${ }^{36}$

Clinical congestion is one of the most common conditions observed in patients with acute decompensated HF. In addition, prolonged congestion leads to longer hospitalization and worse prognoses. ${ }^{7,8}$ Therefore, clinical guidelines suggest rapid evaluation and management of congestion in patients with acute HF. ${ }^{9}$ Medical treatment, including the use of diuretics and/or vasodilators and noninvasive positive pressure ventilation, has been established and should be conducted along a time base. ${ }^{9}$ Although the Efficacy of Vasopressin Antagonism in Heart Failure Outcomes Study with Tolvaptan (EVEREST) trial has identi- fied that residual congestion status at the time of hospital discharge resulted in worse outcomes, ${ }^{10}$ an ideal solution for the treatment of congestion, including the speed of removal during the acute phase after emergency admission in patients with acute decompensated HF, is not likely.

Thus, the present study investigated whether a change in clinical congestion during index hospitalization contributes to improvements in 1-year mortality and rehospitalization rates due to $\mathrm{HF}$ among hospitalized acute decompensated HF patients.

\section{Study Population}

The study population comprised 453 individuals who were hospitalized for acute decompensated HF in Kasugai Municipal Hospital from July 2015 to March 2017. Patients transferred from other hospitals and those who

Received December 16, 2019; revised manuscript received February 20, 2020; accepted March 9, 2020; J-STAGE Advance Publication released online April 21, 2020 Time for primary review: 22 days

Department of Cardiology, Kasugai Municipal Hospital, Kasugai (M.O., K.T., K.Y.); Department of Cardiology, Nagoya University Graduate School of Medicine, Nagoya (H.I., T.T., T.M.); Department of Cardiology, Ichinomiya Municipal Hospital (T.S.); and Division of Medical Statistics, Fujita Health University, Toyoake (H.T.), Japan

The Guest Editor for this article was Dr. Yoshihiko Saito.

Mailing address: Mitsutoshi Oguri, MD, PhD, Department of Cardiology, Kasugai Municipal Hospital, 1-1-1 Takaki-cho, Kasugai 486-8510, Japan. E-mail: oguricap0909@gmail.com

ISSN-1346-9843 All rights are reserved to the Japanese Circulation Society. For permissions, please e-mail: cj@j-circ.or.jp 


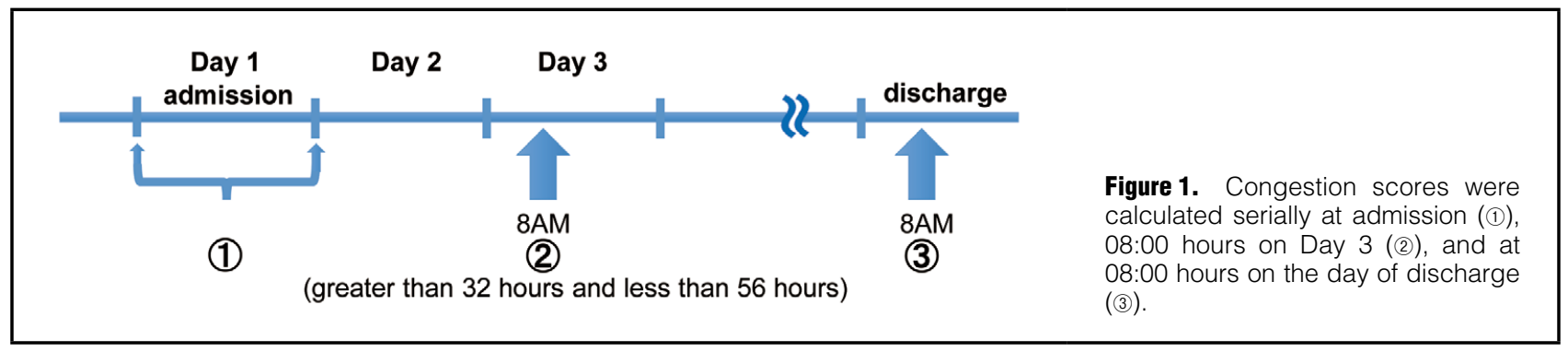

were receiving $\mathrm{HF}$ therapy during hospitalization due to other diseases were excluded from the study. In addition, patients undergoing hemodialysis, those with HF caused by acute myocardial infarction, or those who died within 2 days of hospital admission were excluded. For patients with rehospitalization due to HF within 1 year of the first hospitalization, the first eligible hospitalization was evaluated.

The study protocol complied with the Declaration of Helsinki and was approved by the Committees on Ethics of Kasugai Municipal Hospital (Reference no. 355); the collection of congestion scores was also approved. Each patient was offered the opportunity to opt out of the study (see https://www.hospital.kasugai.aichi.jp/byouin/torikumi/ rinsho/rinri/documents/rinri_6.pdf [in Japanese]). No patient opted-out of the study, so the study was performed on consecutive patients.

\section{Definitions}

$\mathrm{HF}$ was defined according to the American College of Cardiology/American Heart Association guidelines with the signs and symptoms of HF and confirmed left ventricular systolic or diastolic dysfunction. ${ }^{11}$

The congestion score was determined according to the EVEREST trial and consisted of the signs and symptoms of $\mathrm{HF}$, including dyspnea, fatigue, orthopnea, jugular venous distention, rales, and pedal edema. ${ }^{10}$ The composite congestion score was evaluated using a 4-point scale ranging from 0 to 3 for each value and calculated by summing each score. The congestion score was calculated at admission, at 08:00 hours on Day 3 ( $>32$ but $<56$ h after admission), and at 08:00 hours on the day of discharge (Figure 1). The optimal cut-off value for the rate of improvement in congestion scores from admission to Day 3 was determined using the receiver operating characteristic curve method for predicting 1-year mortality.

Anemia was defined as hemoglobin levels $<13 \mathrm{~g} / \mathrm{dL}$ for males and $<12 \mathrm{~g} / \mathrm{dL}$ for females according to World Health Organization (WHO) recommendations. ${ }^{\mathbf{1 2}}$

Causes of death were divided into cardiovascular and non-cardiovascular. Cardiovascular death was defined as any death due to proximate cardiac cause (e.g., myocardial infarction, HF, lethal arrhythmia), whereas non-cardiovascular death was defined as any death from malignancy, respiratory, infectious, or renal causes.

\section{Data Collection}

Clinical characteristics (age, sex, height, weight, previous medical history, New York Heart Association functional classification, etiology of HF, non-cardiac comorbidities, vital signs, and laboratory data), in-hospital treatment, medications at discharge, and 1-year mortality were assessed. Echocardiographic data at discharge were also collected.

\section{Statistical Analysis}

Categorical variables are presented as percentages, whereas continuous variables are presented as the median with interquartile range (IQR) or as the mean \pm SD. Categorical variables were compared by the Chi-squared test. The distribution of continuous variables was examined using the Shapiro-Wilk test; normally distributed variables were compared using the unpaired Student's t-test, whereas variables that were not normally distributed were compared using the Mann-Whitney U-test. Multivariable logistic regression analysis was performed to identify independent predictors of 1-year mortality. Notably, all variables that were statistically significant on univariate analysis were considered as potential covariates: P-values, odds ratios (ORs), and 95\% confidence intervals (CI) were calculated. A P-value of $<0.05$ was considered statistically significant. Statistical significance was examined using 2-sided tests performed in JMP version 13 (SAS Institute, Cary, NC, USA).

\section{Results}

The characteristics of the study subjects $(n=453)$ are given in Table 1. The median age was 81 years (IQR $75-87$ years) and $54.1 \%$ of the subjects were male. The congestion scores at hospital admission, on Day 3, and at discharge were 11 (IQR 8-14), 2 (IQR 1-5), and 0, respectively (Table 2). At the 1-year time point, the cumulative incidence of all-cause mortality was $22.7 \%(n=103): 15.9 \%$ of subjects $(n=72)$ had died from cardiovascular causes, $6.8 \%$ of subjects $(\mathrm{n}=31)$ had died from non-cardiovascular cause, and $20.5 \%(n=93)$ were rehospitalized due to HF (Table 2).

Congestion scores on Day 3 and subjects with residual congestion (defined as those with congestion scores $\geq 3$ on Day 3$)$ were significantly $(\mathrm{P}<0.05)$ greater in subjects who died within 1 year than in those who were alive. However, there were no significant differences in congestion scores at admission and at discharge between these 2 groups. In addition, the rate of improvement in congestion scores $(71.4 \%$ vs. $43.7 \%)$ and the reduction in congestion scores from admission to Day $3(81.3 \%$ vs. $58.3 \%)$ were significantly $(\mathrm{P}<0.05)$ greater in subjects who were alive at 1 year than in those who had died (Table 3).

Univariate analysis revealed that both the congestion score at Day 3 and the rate of improvement in congestion scores from admission to Day 3 were related to 1-year allcause mortality $(\mathrm{P}<0.001)$. The multivariable Cox regression model identified age and left ventricular ejection fraction (LVEF) as significant independent predictors of 


\begin{tabular}{|c|c|}
\hline Age (years) & $81(75-87)$ \\
\hline Males/females (\%) & $54.1 / 45.9$ \\
\hline Current or former smoker (\%) & 47.5 \\
\hline $\mathrm{BMI}\left(\mathrm{kg} / \mathrm{m}^{2}\right)$ & $21.7(19.1-24.6)$ \\
\hline Dyslipidemia (\%) & 22.1 \\
\hline T2D (\%) & 36.2 \\
\hline Hypertension (\%) & 68.6 \\
\hline Reduced $\left(<60 \mathrm{~mL} / \mathrm{min} / 1.73 \mathrm{~m}^{2}\right)$ eGFR $(\%)$ & 78.1 \\
\hline Atrial fibrillation or atrial flutter (\%) & 33.3 \\
\hline Previous MI (\%) & 23.6 \\
\hline Previous stroke (\%) & 12.8 \\
\hline Previous HF hospitalization (\%) & 34.9 \\
\hline Ischemic etiology (\%) & 31.2 \\
\hline \multicolumn{2}{|l|}{ Initial evaluation } \\
\hline $\mathrm{SBP}(\mathrm{mmHg})$ & $147(124-176)$ \\
\hline $\mathrm{DBP}(\mathrm{mmHg})$ & $83(68-102)$ \\
\hline Heart rate (beats/min) & $93(77-115)$ \\
\hline \multicolumn{2}{|l|}{ NYHA functional class (\%) } \\
\hline III & 38.8 \\
\hline IV & 61.2 \\
\hline Jugular venous distension (\%) & 78.2 \\
\hline Blood urea nitrogen (mg/dL) & $25.4(18.7-35.2)$ \\
\hline Sodium (mEq/L) & $140(137-142)$ \\
\hline Potassium (mEq/L) & $4.2(3.8-4.6)$ \\
\hline Creatinine (mg/dL) & $1.21(0.88-1.73)$ \\
\hline Uric acid (mg/dL) & $7.3(5.7-8.8)$ \\
\hline Albumin (mg/dL) & $3.5(3.2-3.8)$ \\
\hline $\mathrm{TC}(\mathrm{mg} / \mathrm{dL})$ & $154(132-180)$ \\
\hline BNP (pg/mL) & $629(310-1,156)$ \\
\hline $\mathrm{Hb}(\mathrm{mg} / \mathrm{dL})$ & $11.2(9.9-13.1)$ \\
\hline $\operatorname{LVEF}(\%)$ & $50(35-64)$ \\
\hline \multicolumn{2}{|l|}{ LVEF categories (\%) } \\
\hline LVEF $<40 \%$ & 31.1 \\
\hline $40 \% \leq \mathrm{LVEF}<50 \%$ & 16.8 \\
\hline LVEF $\geq 50 \%$ & 52.1 \\
\hline
\end{tabular}

Categorical variables are given as percentages, whereas continuous variables are given as the median (interquartile range). $\mathrm{BMI}$, body mass index; BNP, B-type natriuretic peptide; DBP, diastolic blood pressure; eGFR, estimated glomerular filtration rate; $\mathrm{Hb}$, hemoglobin; $\mathrm{HF}$, heart failure; LVEF, left ventricular ejection fraction; MI, myocardial infarction; NYHA, New York Heart Association; SBP, systolic blood pressure; T2D, type 2 diabetes; TC, total cholesterol.

1-year all-cause mortality and cardiovascular mortality (Table 4). Therefore, we compared Kaplan-Meier survival curves for patients based on LVEF using the log-rank test. Patients were divided into three groups based on LVEF $(<40 \%, 40 \% \leq \mathrm{LVEF}<50 \%$, and $\geq 50 \%)$. Event-free survival (either 1-year all-cause mortality or cardiovascular mortality) was significantly higher in subjects with and LVEF $\geq 50 \%$ ( $\mathrm{P}<0.001$; Figure 2). We further examined predictive values by calculating multivariable-adjusted hazard ratios (HRs) for associations of residual congestion (score at Day $3 \geq 3$ ) and the rate of improvement from admission to Day 3 (cut-off value 64\%), and prognostic variables identified by the univariate Cox regression model (age, body mass index, systolic blood pressure, potassium and albumin concentrations, the prevalence of anemia and hypertension,

\begin{tabular}{|lc|}
\hline Table 2. In-Hospital Treatment and Outcomes ( $\mathbf{n}=453)$ \\
In-hospital treatment \\
Intravenous drug therapy \\
$\quad$ Diuretics & $453(96.0)$ \\
Isosorbide dinitrate and/or nitroglycerin & $259(57.2)$ \\
Carperitide & $151(33.4)$ \\
Inotropes & $76(16.7)$ \\
Non-invasive positive pressure ventilation & $97(21.4)$ \\
Medication at discharge & \\
Loop diuretics & $362(88.1)$ \\
Furosemide-equivalent dose (mg) & $40(20-40)$ \\
$\quad \beta$-blocker & $209(50.9)$ \\
$\quad$ ACEl or ARB & $268(65.2)$ \\
$\quad$ Aldosterone blocker & $252(61.3)$ \\
Statin & $135(32.8)$ \\
$\quad$ Nitrates & $37(9.0)$ \\
$\quad$ Antiplatelet drugs & $158(38.4)$ \\
Congestion score (points) & \\
At admission & $11(8-14)$ \\
On hospital Day 3 & $2(1-5)$ \\
At discharge & 0 \\
Hospital length of stay (days) & $16(11-24)$ \\
1-year outcomes & \\
Mortality & $103(22.7)$ \\
Cardiovascular mortality & $72(15.9)$ \\
Non-cardiovascular mortality & $31(6.8)$ \\
Rehospitalization due to heart failure & $93(20.5)$ \\
\hline
\end{tabular}

Categorical variables are given as percentages, whereas continuous variables are given as the median (interquartile range). $\mathrm{ACEl}$, angiotensin-converting enzyme inhibitor; $\mathrm{ARB}$, angiotensin II receptor blocker.

LVEF, ischemic etiology, and previous HF hospitalization). Relative risk of 1-year all-cause mortality was higher in subjects with residual congestion and lesser improvement $(<64 \%)$ than in those with residual congestion and greater improvement ( $\geq 64 \%$; adjusted [a] HR $2.33,95 \%$ CI $1.11-4.91, \mathrm{P}=0.025$ ) or resolved congestion (score at Day $3<3$; aHR 2.17, 95\% CI 1.30-3.63, P=0.003; Table 5; Figure 3). Similar analysis revealed a significant relationship between cardiovascular mortality and both congestion score at Day 3 and the rate of improvement from admission to Day 3. Combined predictive values of residual congestion and lesser improvement with adjustment for prognostic variables identified by the univariate Cox regression model (age, body mass index, systolic blood pressure, potassium and albumin concentrations, the prevalence of anemia, reduced estimated glomerular filtration rate [eGFR], and hypertension, LVEF, ischemic etiology, and previous HF hospitalization) were higher than those for residual congestion and higher improvement (aHR $3.04,95 \%$ CI $1.15-8.03, \mathrm{P}=0.025)$, or resolved congestion (aHR 3.17, 95\% CI 1.65-6.11, P<0.001; Table 5). Multivariate Cox regression analysis revealed that age, serum potassium, the prevalence of anemia and reduced eGFR, LVEF, and previous HF hospitalization were significantly and independently associated with rehospitalization due to HF. However, there was no relationship between the congestion score at Day 3 or the rate of improvement from admission to Day 3 and rehospitalization due to $\mathrm{HF}$. 


\begin{tabular}{|c|c|c|c|c|}
\hline & $\begin{array}{l}\text { All patients } \\
(n=453)\end{array}$ & $\begin{array}{l}\text { Dead at } 1 \text { year } \\
\quad(n=103)\end{array}$ & $\begin{array}{l}\text { Alive at } 1 \text { year } \\
\quad(n=350)\end{array}$ & P-value ${ }^{A}$ \\
\hline \multicolumn{5}{|l|}{ Congestion score } \\
\hline At admission (points) & $11(8-14)$ & $11(8-14)$ & $11(8-14)$ & 0.346 \\
\hline On Day 3 (points) & $2(1-5)$ & $4(1-7)$ & $2(0-4)$ & $<0.001^{*}$ \\
\hline$\geq 3$ points $(\%)$ & 46.6 & 66.0 & 40.9 & $<0.001^{*}$ \\
\hline At discharge (points) & 0 & $0(0-1)$ & 0 & 0.065 \\
\hline$\geq 3$ points $(\%)$ & 3.9 & 4.9 & 3.7 & 0.654 \\
\hline$\%$ Reduction in scores ${ }^{B}$ & $77.8(50.0-92.9)$ & $58.3(33.3-85.7)$ & $81.3(571-100)$ & $<0.001^{*}$ \\
\hline Reduction in scores ${ }^{B}$ (points) & $7(4-11)$ & $6(4-9)$ & $8(4-11)$ & $0.009^{*}$ \\
\hline Rate of improvement ${ }^{\mathrm{B}}(\%)$ & 65.1 & 43.7 & 71.4 & $<0.001^{*}$ \\
\hline
\end{tabular}

Continuous variables are given as the median (interquartile range). AP-values are for comparisons between subjects who were alive at 1 year and those who had died. ${ }^{B}$ From admission to Day 3 . ${ }^{*} \mathrm{P}<0.05$.

\begin{tabular}{|c|c|c|c|c|}
\hline \multirow[b]{3}{*}{ All-cause mortality } & \multicolumn{2}{|c|}{ Univariate } & \multicolumn{2}{|c|}{ Multivariate } \\
\hline & HR $(95 \% \mathrm{Cl})$ & P-value & $\mathrm{HR}(95 \% \mathrm{Cl})$ & P-value \\
\hline & & & & \\
\hline Age & $1.05(1.03-1.07)$ & $<0.001^{*}$ & $1.06(1.03-1.09)$ & $<0.001^{*}$ \\
\hline $\mathrm{BMI}$ & $0.91(0.86-0.95)$ & $<0.001^{*}$ & & 0.151 \\
\hline SBP & $0.99(0.99-1.00)$ & $0.001^{*}$ & & 0.498 \\
\hline Potassium & $1.45(1.15-1.79)$ & $0.002^{*}$ & & 0.372 \\
\hline Albumin & $0.53(0.37-0.77)$ & $0.001^{*}$ & & 0.057 \\
\hline Anemia & $1.76(1.34-2.41)$ & $<0.001^{*}$ & & 0.065 \\
\hline LVEF & $0.97(0.95-0.98)$ & $<0.001^{*}$ & $0.96(0.94-0.98)$ & $<0.001^{*}$ \\
\hline Ischemic & $1.52(1.25-1.84)$ & $<0.001^{*}$ & & 0.103 \\
\hline Previous HF hospitalization & $1.33(1.09-1.61)$ & $0.004^{*}$ & & 0.137 \\
\hline Hypertension & $0.70(0.58-0.85)$ & $<0.001^{*}$ & & 0.147 \\
\hline \multicolumn{5}{|l|}{ Cardiovascular mortality } \\
\hline Age & $1.05(1.02-1.08)$ & $<0.001^{*}$ & $1.06(1.02-1.10)$ & $0.006^{*}$ \\
\hline BMI & $0.91(0.85-0.97)$ & $0.002^{*}$ & & 0.240 \\
\hline SBP & $0.99(0.98-1.00)$ & $0.002^{*}$ & & 0.346 \\
\hline Potassium & $1.63(1.26-2.05)$ & $<0.001^{*}$ & & 0.238 \\
\hline Albumin & $0.53(0.35-0.83)$ & $0.005^{*}$ & & 0.137 \\
\hline Anemia & $4.10(1.88-8.95)$ & $<0.001^{*}$ & & 0.119 \\
\hline Reduced eGFR & $3.15(1.37-7.27)$ & $0.007^{*}$ & & 0.197 \\
\hline LVEF & $0.97(0.95-0.98)$ & $<0.001^{*}$ & $0.96(0.94-0.98)$ & $<0.001^{*}$ \\
\hline Ischemic & $2.40(1.51-3.81)$ & $<0.001^{*}$ & & 0.524 \\
\hline Previous HF hospitalization & $1.97(1.24-3.13)$ & $0.004^{*}$ & & 0.113 \\
\hline Hypertension & $0.50(0.31-0.79)$ & $0.003^{*}$ & & 0.208 \\
\hline
\end{tabular}

$\mathrm{Cl}$, confidence interval; HR, hazard ratio. Other abbreviations as in Table 1. ${ }^{*} \mathrm{P}<0.05$.

Given the possibility of B-type natriuretic peptide (BNP)-guided therapy, we evaluated BNP concentrations across the 3 groups (Supplementary Table). The median admission BNP concentration (pg/mL) was 599 (IQR 2591,046), 627 (IQR 316-1,033), and 694 (IQR 415-1,482) for patients with resolved congestion, residual congestion with improvement, and residual congestion without improvement, respectively $(\mathrm{P}=0.013)$. Conversely, the median predischarge BNP concentration was not significantly different among patients with resolved congestion, residual congestion with improvement, and residual congestion without improvement (259 [IQR 123-484], 247 [IQR 113484], and 345 [IQR 152, 561] pg/mL, respectively). We also evaluated other variables, including congestion scores and their constituents (Supplementary Table). Age and serum concentrations of blood urea nitrogen, creatinine, and BNP were greater in subjects with residual congestion and lesser improvement than in those with resolved congestion or residual congestion and higher improvement. However, the ratio of current or former smokers, the prevalence of hypertension, systolic blood pressure, and serum albumin, total cholesterol, and hemoglobin concentrations were lower in subjects with residual congestion and lesser improvement.

Serial measurements in eGFR during first 3 days were available for 376 patients ( $83.0 \%$ of study subjects). The decline in eGFR $>20 \%$ was slightly greater in subjects with residual congestion than in those with removed congestion 
(A)

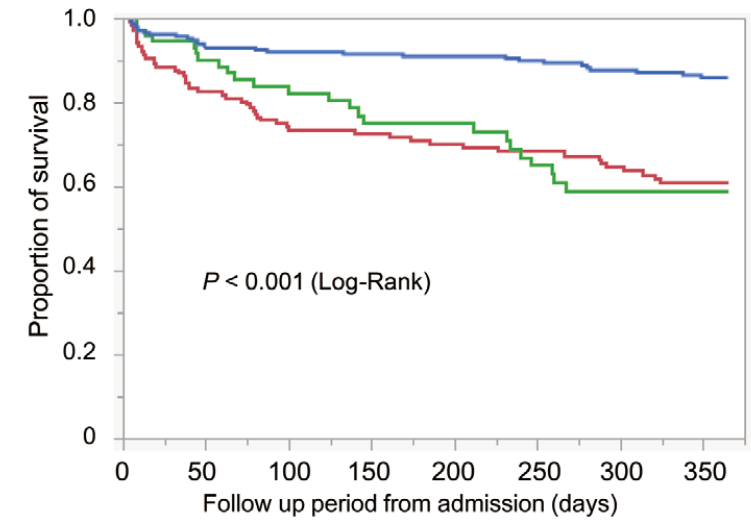

(B)

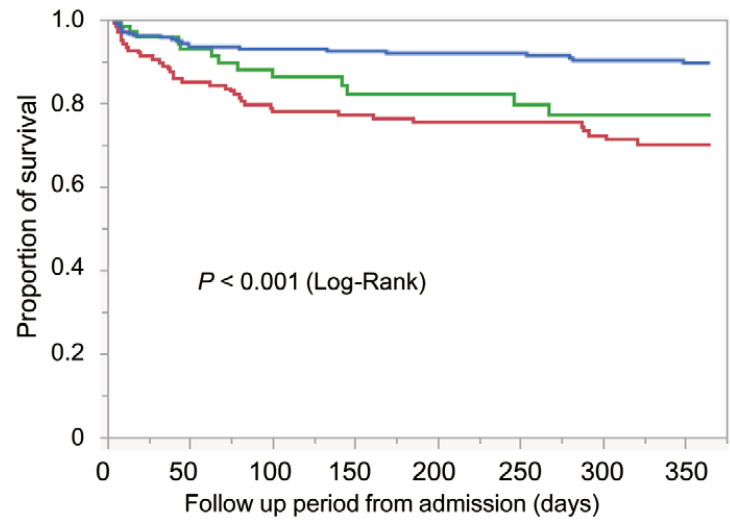

\begin{tabular}{|c|c|c|c|c|c|c|c|c|c|c|c|c|c|c|c|}
\hline \multicolumn{9}{|c|}{ Number at risk } & \multicolumn{7}{|c|}{ Number at risk } \\
\hline LVEF $\geq 50 \%$ & 220 & 218 & 217 & 216 & 214 & 211 & 208 & LVEF $\geq 50 \%$ & 221 & 220 & 219 & 218 & 218 & 215 & 214 \\
\hline $40 \% \leq \mathrm{LVEF}<50 \%$ & 69 & 64 & 60 & 60 & 55 & 52 & 52 & $40 \% \leq \mathrm{LVEF}<50 \%$ & 71 & 67 & 65 & 65 & 64 & 63 & 63 \\
\hline LVEF $<40 \%$ & 125 & 113 & 112 & 109 & 107 & 103 & 99 & LVEF $<40 \%$ & 121 & 112 & 111 & 109 & 109 & 106 & 104 \\
\hline
\end{tabular}

Figure 2. Survival curves for (A) 1-year all-cause mortality and (B) cardiovascular mortality according to left ventricular ejection fraction (LVEF).

Table 5. Predictive Value of Congestion Scores on Day 3 and Improvement in Congestion Scores for 1-Year Mortality and Rehospitalization Due to HF According to Cox Regression Analysis

\begin{tabular}{|c|c|c|c|c|}
\hline & \multicolumn{2}{|c|}{ Not adjusted } & \multicolumn{2}{|c|}{ AdjustedA } \\
\hline & HR (95\% Cl) & P-value & HR (95\% Cl) & P-value \\
\hline \multicolumn{5}{|l|}{ All-cause mortality } \\
\hline Score on Day $3 \geq 3$ points & $2.68(1.78-4.03)$ & $<0.001^{*}$ & $1.76(1.07-2.80)$ & $0.024^{*}$ \\
\hline Improvement $<64 \%$ & $3.04(2.06-4.49)$ & $<0.001^{*}$ & $2.21(1.37-3.57)$ & $0.001^{*}$ \\
\hline Score on Day $3<3$ points & Ref. & $<0.001^{\mathrm{B} *}$ & Ref. & $<0.001^{\mathrm{B} *}$ \\
\hline Score on Day $3 \geq 3$ points with $\geq 64 \%$ improvement & $1.23(0.64-2.50)$ & 0.496 & $0.93(0.44-1.97)$ & 0.848 \\
\hline Score on Day $3 \geq 3$ points with $<64 \%$ improvement & $3.21(2.10-4.90)$ & $<0.001^{*}$ & $2.17(1.30-3.63)$ & $0.003^{*}$ \\
\hline \multicolumn{5}{|l|}{ Cardiovascular mortality } \\
\hline Score on Day $3 \geq 3$ points & $3.76(2.22-6.35)$ & $<0.001^{*}$ & $2.41(1.29-4.50)$ & $0.006^{*}$ \\
\hline Improvement $<64 \%$ & $4.31(2.65-7.01)$ & $<0.001^{*}$ & $3.14(1.72-5.74)$ & $<0.001^{\star}$ \\
\hline Score on Day $3<3$ points & Ref. & $<0.001^{\mathrm{B} *}$ & Ref. & $<0.001^{B *}$ \\
\hline Score on Day $3 \geq 3$ points with $\geq 64 \%$ improvement & $1.51(0.63-3.62)$ & 0.354 & $1.04(0.38-2.88)$ & 0.933 \\
\hline Score on Day $3 \geq 3$ points with $<64 \%$ improvement & $4.76(2.76-8.21)$ & $<0.001^{*}$ & $3.17(1.65-6.11)$ & $<0.001^{*}$ \\
\hline \multicolumn{5}{|l|}{ Heart failure readmission } \\
\hline Score on Day $3 \geq 3$ points & & 0.148 & & \\
\hline Improvement $<64 \%$ & & 0.467 & & \\
\hline
\end{tabular}

AFor all-cause mortality, adjusted for age, BMI, SBP, potassium and albumin concentrations, the prevalence of anemia and hypertension, LVEF, ischemic etiology, and previous HF hospitalization; for cardiovascular mortality, adjusted for age, BMI, SBP, potassium and albumin concentrations, the prevalence of anemia, hypertension, and reduced eGFR, LVEF, ischemic etiology, and previous HF hospitalization. These covariates were found to have $\mathrm{P}<0.05$ by univariate Cox regression analysis (see Table 4). BP-values for trend. Abbreviations as in Tables 1,4 . ${ }^{*} \mathrm{P}<0.05$.

(18.1\% vs. $17.6 \%)$, but the difference was not statistically significant $(\mathrm{P}=0.893)$. Multivariable logistic regression analysis revealed that albumin concentration and the prevalence of anemia were significantly and independently associated with the presence of residual congestion (ORs 1.60 [95\% CI-1.02-2.49; $\mathrm{P}=0.002]$ and 0.63 [95\% CI 0.40 $0.98 ; \mathrm{P}=0.040]$, respectively).

\section{Discussion}

The results of the present study indicate that residual congestion at Day 3 or lesser improvement from admission to Day 3 are both associated with increased 1-year mortality in patients with acute decompensated HF. Furthermore, both residual congestion and lesser improvement in congestion scores become prognostic values after adjusting for 


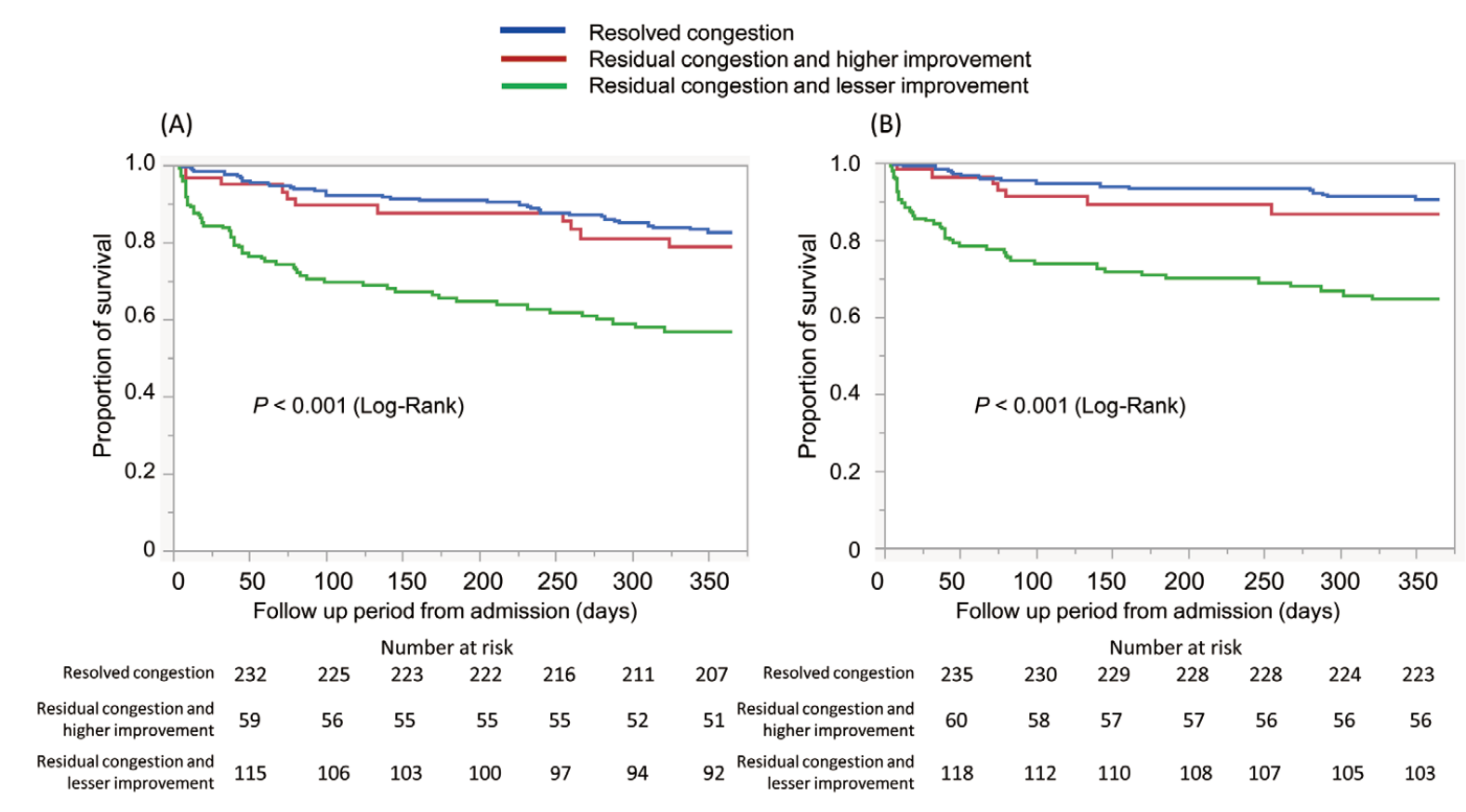

Figure 3. Survival curves for (A) 1-year all-cause mortality and (B) cardiovascular mortality according to congestion status: resolved congestion, residual congestion with greater improvement, and residual congestion with lesser improvement.

other existing clinical factors.

We also identified that rapid congestion improvement in patients was associated with a favorable prognoses, even if these patients had residual congestion on Day 3. Congestion has been shown to be associated with troponin levels and may lead directly to subclinical myocardial injury. ${ }^{\mathbf{1 3}}$ Furthermore, subsequent pathways from congestion leading to adverse outcomes include elevated venous pressures that compromise the function of the kidney or other organs. ${ }^{14}$ Therefore, a rapid improvement in clinical signs, such as dyspnea, is desirable in patients hospitalized with acute decompensated HF. The results of this study may reflect the guideline-recommended and established strategy, consistent with prior observations. However, it is uncertain that the rapid relief of congestion, regardless of residual congestion, offers a favorable prognosis.

Clinical congestion was reported to be an important therapeutic target of management in the EVEREST trial.15 We recognize that the congestion score proposed in the EVEREST trial may be beneficial from the viewpoint of both subjective and objective values; thus, we determined this score serially in the present study. A composite congestion score at discharge or Day 7 has been identified as being independently associated with prognosis; ${ }^{10}$ however, serial changes during the acute phase during hospitalization were not described in that study. The comparison of results between the present study and the EVEREST trial is difficult because of differences in the severity of the index congestion (initial median congestion score 11 points in the present study vs. 4.1 points in the EVEREST trial), and the score at discharge (almost 0) was not associated with 1 -year mortality in the present study, which may be due to the longer hospitalization period (median 16 days; IQR 11-24 days). Pang et al proposed a tool to evaluate conges- tion in patients with acute decompensated HF, namely the Provocative Dyspnea Assessment, a measurement method that combines sequential dyspnea provocation by positioning and walking. ${ }^{16}$ Pang et al also noted the need to measure symptom severity at each time point using the same measurement. ${ }^{16}$ However, it is difficult to use this assessment tool with all HF patients in real-world clinical practice. In addition, congestion assessment using the radiographic congestion score index has been important in predicting mid-term prognosis in HF patients. ${ }^{17}$ This approach could be beneficial, but it is possible that there may be limited evidence in this comprehensive design. Although the predischarge BNP concentration is an established prognostic marker for predicting the post-discharge cardiac events, ${ }^{18}$ this value did not match the relationship identified in the present study.

The present study has some limitations. First, the results presented here are only from a single hospital and the sample size is relatively small. Second, we evaluated congestion status only using the investigator-assessed congestion score, and did not take patient-reported aspects into account. Therefore, validation of the findings using other congestion tools will be necessary. Third, patients who died within 2 days of hospital admission were excluded from the study; thus, the present results cannot be extrapolated to the most critically ill or severely decongested patients. Finally, the relationship between rapid decongestion therapy and known long-term clinical factors (cardiac troponin or serial changes in eGFR) was not confirmed.

In conclusion, this study suggests that rapid decongestion therapy after hospital admission could be prerequisite for improving 1-year mortality regardless of whether residual congestion presents in acute decompensated HF. 


\section{Acknowledgment}

The authors are grateful to the staff at Kasugai Municipal Hospital for their assistance collecting the medical data.

\section{Conflict of Interest}

T.M. is a member of Circulation Journal' Editorial Team. The other authors declare no conflicts of interest.

\section{Data Availability}

Deidentified participant data will be shared on request to the corresponding author. All data will be shared, as will the study protocol. Data will be available from within 6 months after publication for a period of 1 year. Data will be shared after permission has been granted by the Committees on Ethics of Kasugai Municipal Hospital.

\section{References}

1. Okura Y, Ramadan MM, Ohno Y, Mitsuma W, Tanaka K, Ito $\mathrm{M}$, et al. Impending epidemic: Future projection of heart failure in Japan to the year 2055. Circ J 2008; 72: 489-491.

2. Ushigome R, Sakata Y, Nochioka K, Miyata S, Miura M, Tadaki S, et al. Temporal trends in clinical characteristics, management and prognosis of patients with symptomatic heart failure in Japan: Report from the CHART Studies. Circ J 2015; 79: $2396-2407$.

3. Dharmarajan K, Rich MW. Epidemiology, pathophysiology, and prognosis of heart failure in older adults. Heart Fail Clin 2017; 13: 417-426.

4. Gheorghiade M, Vaduganathan M, Fonarow GC, Bonow RO. Rehospitalization for heart failure: Problems and perspectives. $J$ Am Coll Cardiol 2013; 61: 391-403.

5. Moita B, Marques AP, Camacho AM, Leão Neves P, Santana $\mathrm{R}$. One-year rehospitalisations for congestive heart failure in Portuguese NHS hospitals: A multilevel approach on patterns of use and contributing factors. BMJ Open 2019; 9: e031346.

6. O'Connor CM, Miller AB, Blair JE, Konstam MA, Wedge P, Bahit MC, et al; Efficacy of Vasopressin Antagonism in heart Failure Outcome Study with Tolvaptan (EVEREST) investigators. Causes of death and rehospitalization in patients hospitalized with worsening heart failure and reduced left ventricular ejection fraction: Results from Efficacy of Vasopressin Antagonism in Heart Failure Outcome Study with Tolvaptan (EVEREST) program. Am Heart J 2010; 159: 841-849.

7. Gheorghiade M, Filippatos G, De Luca L, Burnett J. Congestion in acute heart failure syndromes: An essential target of evaluation and treatment. Am J Med 2006; 119(Suppl 1): S3-S10.

8. Goldsmith SR, Brandimarte F, Gheorghiade M. Congestion as a therapeutic target in acute heart failure syndromes. Prog Cardiovasc Dis 2010; 52: 383-392.

9. Ponikowski P, Voors AA, Anker SD, Bueno H, Cleland JG,
Coats AJ, et al. 2016 ESC guidelines for the diagnosis and treatment of acute and chronic heart failure: The Task Force for the Diagnosis and Treatment of Acute and Chronic Heart Failure of the European Society of Cardiology (ESC). Developed with the special contribution of the Heart Failure Association (HFA) of the ESC. Eur J Heart Fail 2016; 18: 891-975.

10. Ambrosy AP, Pang PS, Khan S, Konstam MA, Fonarow GC, Traver B, et al; EVEREST Trial Investigators. Clinical course and predictive value of congestion during hospitalization in patients admitted for worsening signs and symptoms of heart failure with reduced ejection fraction: Findings from the EVEREST trial. Eur Heart J 2013; 34: 835-843.

11. Yancy CW, Jessup M, Bozkurt B, Butler J, Casey DE Jr, Colvin MM, et al. 2017 ACC/AHAHFSA focused update of the 2013 ACCF/AHA guideline for the management of heart failure: A report of the American College of Cardiology/American Heart Association Task Force on Clinical Practice Guidelines and the Heart Failure Society of America. Circulation 2017; 136: e137-e161.

12. Nutritional anaemias: Report of a WHO scientific group. World Health Organ Tech Rep Ser 1968; 405: 5-37.

13. Negi S, Sawano M, Kohsaka S, Inohara T, Shiraishi Y, Kohno $\mathrm{T}$, et al. Prognostic implication of physical signs of congestion in acute heart failure patients and its association with steady-state biomarker levels. PLoS One 2014; 9: e96325.

14. Mullens W, Abrahams Z, Francis GS, Sokos G, Taylor DO, Starling RC, et al. Importance of venous congestion for worsening of renal function in advanced decompensated heart failure. $J$ Am Coll Cardiol 2009; 53: 589-596.

15. Gheorghiade M, Konstam MA, Burnett JC Jr, Grinfeld L, Maggioni AP, Swedberg K, et al. Short-term clinical effects of tolvaptan, an oral vasopressin antagonist, in patients hospitalized for heart failure: The EVEREST Clinical Status Trials. JAMA 2007; 297: 1332-1343.

16. Pang PS, Cleland JG, Teerlink JR, Collins SP, Lindsell CJ, Sopko G, et al; Acute Heart Failure Syndromes International Working Group. A proposal to standardize dyspnoea measurement in clinical trials of acute heart failure syndromes: The need for a uniform approach. Eur Heart J 2008; 29: 816-824.

17. Kobayashi M, Watanabe M, Coiro S, Bercker M, Paku Y, Iwasaki $\mathrm{Y}$, et al. Mid-term prognostic impact of residual pulmonary congestion assessed by radiographic scoring in patients admitted for worsening heart failure. Int J Cardiol 2019; 289: 91-98.

18. Logeart D, Thabut G, Jourdain P, Chavelas C, Beyne P, Beauvais $\mathrm{F}$, et al. Predischarge B-type natriuretic peptide assay for identifying patients at high risk of re-admission after decompensated heart failure. J Am Coll Cardiol 2004; 43: 635-641.

\section{Supplementary Files}

Please find supplementary file(s);

http://dx.doi.org/10.1253/circj.CJ-19-1128 